\title{
Chaotic Genetic Algorithm based on Lorenz Chaotic System for Optimization Problems
}

\author{
Reza Ebrahi mzadeh \\ Department of Computer Science, Zahedan Branch, Islamic Azad University, Zahedan, Iran \\ Email: Ebrahimzade@iauzah.ac.ir \\ Mahdi Jampour (Correspondence Author) \\ Institute for Computer Graphics and Vision, Graz University of Technology, Graz, Austria \\ Email: Jampour@IEEE.org
}

\begin{abstract}
Very recently evolutionary optimization algorithms use the Genetic Algorithm to improve the result of Optimization problems. Several processes of the Genetic Algorithm are based on 'Random', that is fundamental to evolutionary algorithms, but important defections in the Genetic Algorithm are local convergence and high tolerances in the results, they have happened for randomness reason. In this paper we have prepared pseudo random numbers by Lorenz chaotic system for operators of Genetic Algorithm to avoid local convergence. The experimental results show that the proposed method is much more efficient in comparison with the traditional Genetic Algorithm for solving optimization problems.
\end{abstract}

Index Terms - Optimization Algorithm, Chaos Genetic Algorithm, Evolutionary Algorithm, Schaffer, Clonalg

\section{Introduction}

\subsection{Review}

Nowadays, nonlinear optimization problems are one of the most important problems in computational theory. Because many of applications in the real world need to be optimize. There are many techniques to solve optimization problems and they also have been tried to improve their important measures such as time complexity. On the other hand there are many evaluation functions and benchmarks to evaluate optimization techniques and researchers are using them to evaluate their techniques and methods, the most famous of these functions are Schaffer, Clonalg, Bohachevsky, Rosenbrock, TSP (Travelling Sales man Problem), etc. Hyper-heuristic techniques and Evolutionary algorith ms are two solutions for nonlinear optimization problems. The Genetic Algorithm is an evolutionary algorithm that can solve these problems. Evolutionary algorithms have many benefits in solving problems but they have a few disadvantages, one of the disadvantages is 'premature convergence' that is for
Random reason. Some evolutionary algorithms are GA, PSO, SA, ACO, etc. Many researchers introduced new improved methods based on the above techniques; Tsoulos [1] introduced a heuristic modified method based on the Genetic Algorithm. In that paper he modified genetic operators that preserve the feasibility of the trial solution encoded in the chromosomes. Also, there are hybrid algorithms to solve optimization problems; most of them trying to improve other important parameters such as time complexity, adaptability, stability, etc. Wei Juan [2] and his coauthor optimized the Fuzzy Rule Base with combination of the Genetic Algorithm and Ant Colony; their results show that the hybrid method can be more useful than the basic Genetic Algorithm. In another study, Sun Feng-jie [3] and his coauthor proposed an efficient hybrid method for image classification with PSO and GA; they used features of fast convergence of particle swarm optimization and diversity of genetic algorithm to improve their method.

\subsection{Motivation for Using Chaotic Systems}

Evaluation of Genetic Algorithms in optimization problems in previous studies have shown that premature convergence is a disadvantage to solve these problems; this is for randomness reason that use in Initial Population, Crossover and Mutation processes. Our experience says, determin istic pseudo-random data can be useful for diversity of Chromosomes and finally in the results. In fact pseudo-randomness and deterministic are two features of chaotic systems that are interesting in this paper. Since our research was shown that the Loren $z$ chaotic systems outperforms these other systems, we use it in the present research.

\subsection{The-State-of-the-Art}

Recently, use of the Chaos Theory and chaotic values has increased significantly, many of applications in different fields of nonlinear science and also computer science have employed Chaos Theory and achieved its 
benefits, such as Encryption algorithms that use a chaotic map to create secure images in image encryption techniques [4, 5, 6 and 7], using chaotic systems in Time Series Predictions [8 and 9] and Communication systems [10], for Clustering that Chang and Huang [11] proposed a chaos clustering approach based on Genetic Algorithm in their contribution and achieved both enhance to diversity and extract clustering rules for achieving a potential moving track of evolution to improve the convergence performance. Also in Optimization Problems [12 and 13] where Gandomi and his coauthors in [12] proposed a Chaotic Accelerated PSO that can outperform standard APSO with appropriate performance in comparison with other algorithms and in application to a complex problem. Most of the optimization methods which use chaotic systems instead of random processes trying to generate deterministic data with random values. The Genetic Algorithm, Particle Swarm Optimization and other Evolutionary Algorithms are some of the methods recently use chaotic systems.

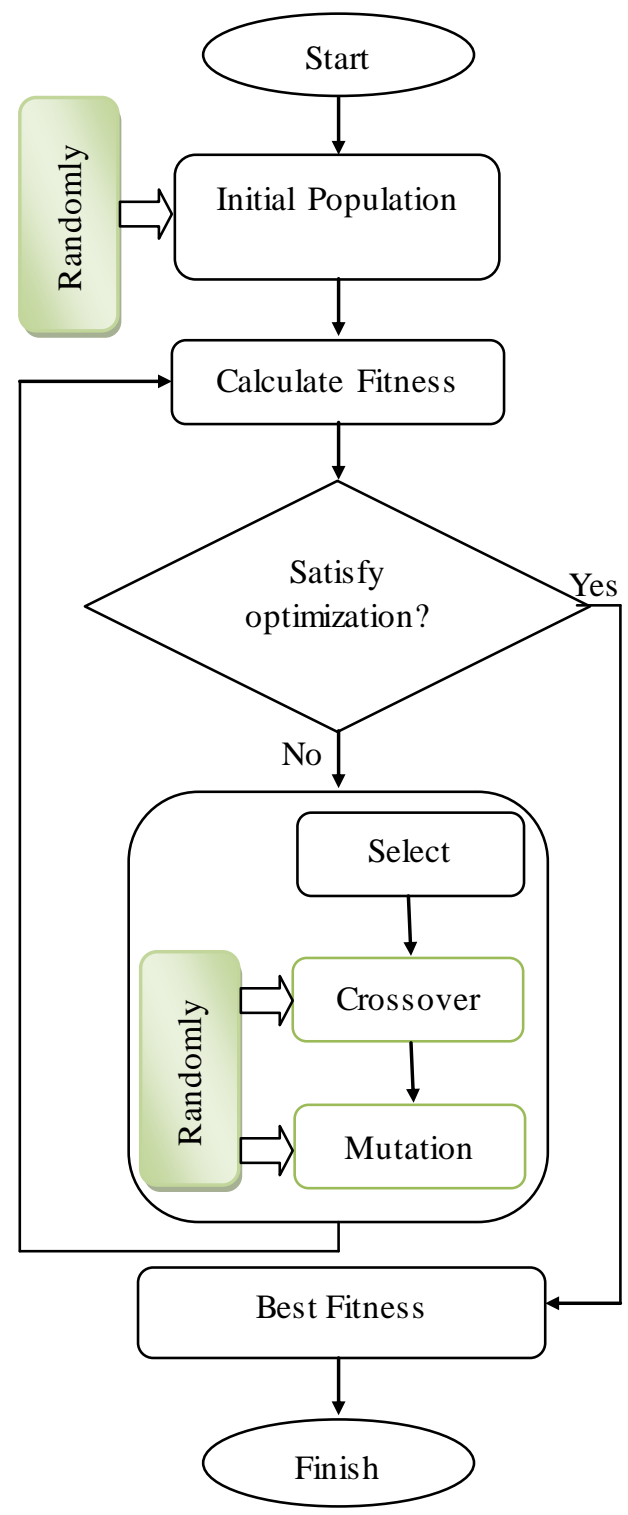

Fig. 1: Basic Genetic Algorithm flowchart

\section{Basic Genetic Algorithm}

The Genetic Algorithm was introduced by Professor John Holland in the 1970s who took advantage of some phenomenon in natural evolution; he introduced his idea based on Darwin's Biological Evaluation as an optimization algorithm. The main idea in this technique is derived from natural evolution so there are some biological operators such as Crossover, Mutation and Selection [14]. There are three part of this process which are randomly generated, namely the Initial Population, the Crossover and the Mutation. In the first step of the process, the Initial Population in the Genetic Algorithm generates some random solutions. In the second step, the random value of Crossover aid to make new offspring and in the third step, with random value of Mutation let to change a few of gens [15]. Figure 1 shows a flowchart of the basic Genetic Algorithm idea. Where there are some important sections such as Initial Population, Fitness evaluation, Crossover and Mutation. In general, the Genetic Algorithm strictly depends on Crossover and Mutation operators; this means that the Random function is very important in the Genetic Algorithm. The Genetic Algorithm has desirable time complexity, but it can still be improved further.

\section{Chaotic Systems}

The idea of using chaotic systems instead of a random process has been noticed in optimization problems. Chaos is a common non-linear phenomenon in nature, which fully reflects the complexity of the system. Chaos is random and has a clutter performance like a random variable [16]; in fact the rule of randomness can be played by chaotic dynamics instead of a random process [17]. Chaotic systems include features that can be important in optimization problems, such as:

- The sensitive dependence on initial conditions;

- The quasi-stochas tic property;

- Ergodicity.

Implementation and Analysis of optimization based on chaotic systems in this paper show that the above features are important and they can improve the efficiency of the Genetic Algorithm in solving computational problems.

$$
\begin{aligned}
& \dot{x}=10(y-x) \\
& \dot{y}=28 x-x z+y \\
& \dot{z}=x y-\frac{8}{3} z
\end{aligned}
$$

\subsection{Lorenz Chaotic Systems}

The Lorenz system is one of the famous chaotic systems that it was introduced by Edward Lorenz. Loren $z$ system equations were introduced in equation 
(1); the Lorenz system is a 3 dimensional system and its lyapunov exponents have been shown in figure 2 , where three exponents are $\lambda_{1}=1.06 \cdot \lambda_{2}=0$ and $\lambda_{3}=-12.7$ $[18,19]$.
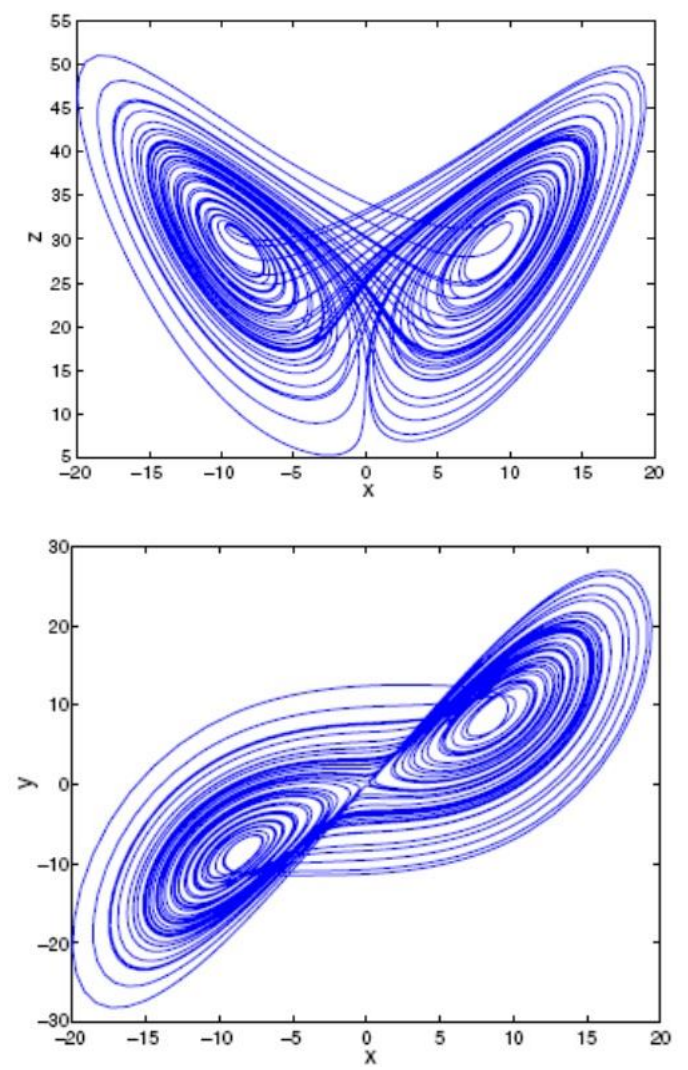

Fig. 2: Lorenz system's lyapunov exponents
According to the Lorenz chaotic system in equation (1), its output has been generated and compared with random values. Figure 3 shows the comparis on between random values and Loren $z$ chaotic output values. As we generated 10,000 rando $m$ and chaos numbers between 1 and 10; it is obvious the count of each numbers must be $1000 \pm \alpha$, which the diversity of values in Genetic Algorithm mechanism will be better than if $\alpha \rightarrow 0$. On the other hand, the local convergence that is a disadvantage of traditional the Genetic Algorithm will happen if the value of $\alpha$ is growth.

\section{Chaotic Genetic Algorithm}

Genetic Algorithm uses random processes in Initial population, Crossover and Mutation process that they can be replaced with chaotic systems such as Lorenz or other chaotic systems such as Hennon map, Logistic map, etc. Pseudo-randomness and ergodicity are two dynamic characteristic of chaotic structures and they are the reason of using chaos systems in Genetic Algorithm instead random processes because they will avoid local convergence. The flowchart of proposed Chaotic Genetic Algorithm has been shown in figure 4; in proposed method we replace random processes in Initial population, Crossover and Mutation with Lorenz chaotic output.

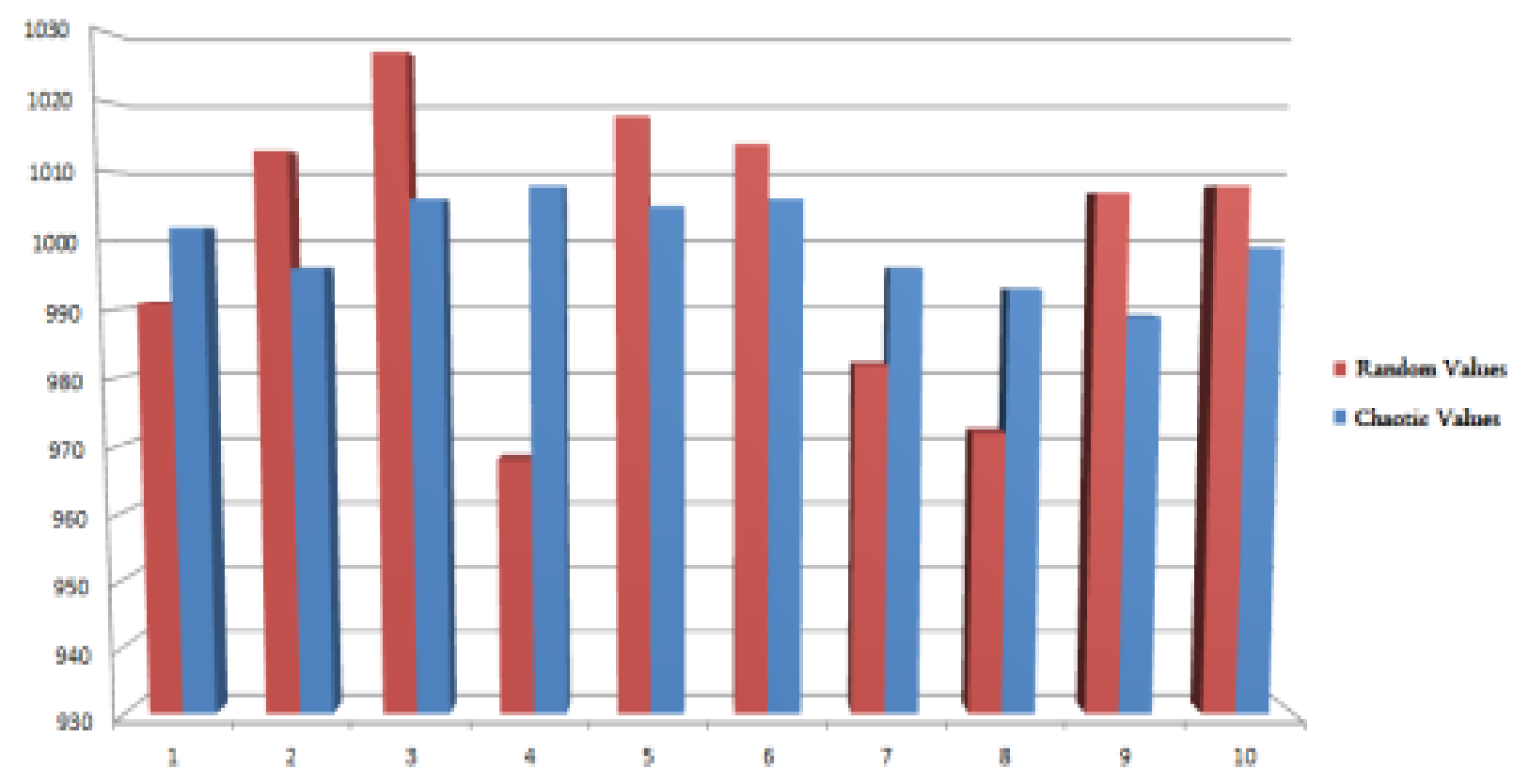

Fig. 3: The frequency charts of 10,000 generated Random and Chaotic numbers 


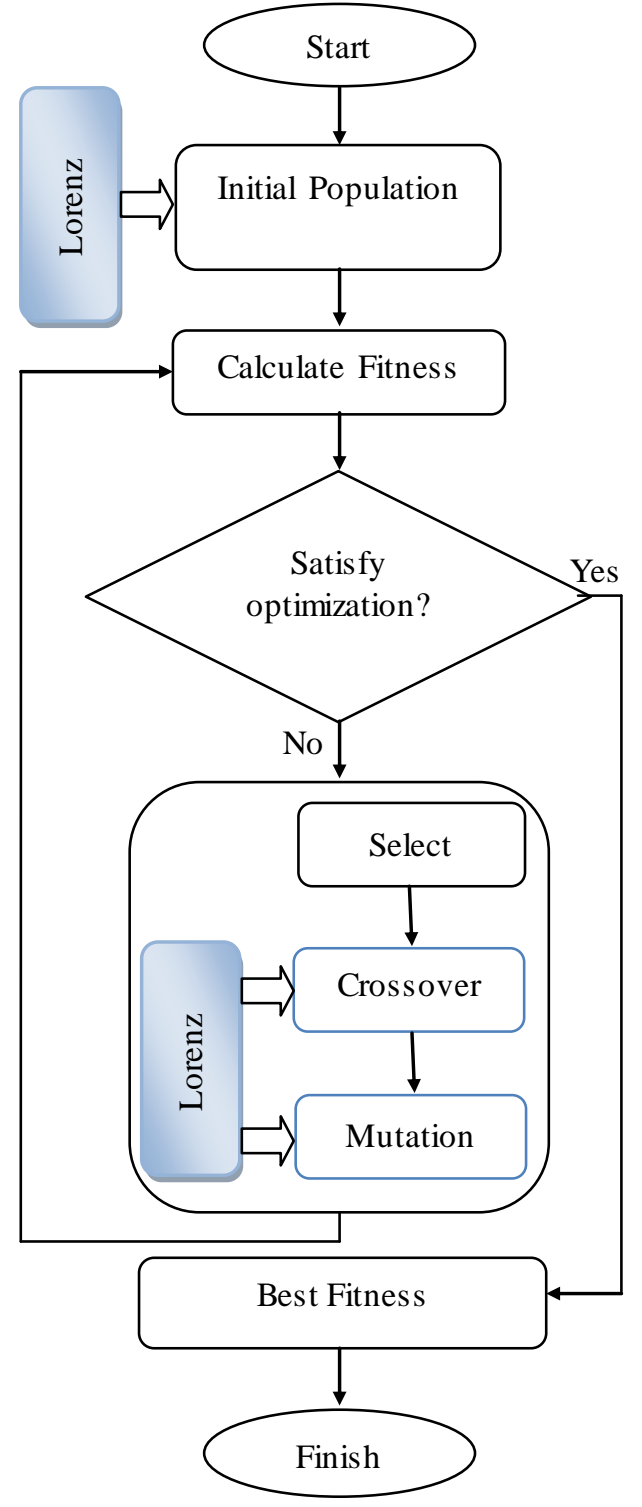

Fig. 4: Chaotic Genetic Algorithm flowchart

\section{Chaotic Genetic algorithm and Benchmarks}

We implement the Chaos Genetic Algorithm based on the Lorenz chaotic system in MATLAB and test on some famous benchmarks such as Schaffer and Clonalg functions. Schaffer is introduced in equation (2) and also we introduce Clonalg in equation (3). These two functions have many good features for optimization problems such as kind of acceptable optimums. These functions have been shown in figure 5 .

$$
\begin{aligned}
& f(x, y)=0.5-\frac{\sin ^{2} \sqrt{x^{2}+y^{2}}-0.5}{\left(1+0.001\left(x^{2}+y^{2}\right)\right)^{2}} \quad x, y \in[-100,100] \\
& f(x, y)=x \sin (4 \pi x)-y \sin (4 \pi y+\pi)+1 \quad x, y \in[-200,200]
\end{aligned}
$$

The Schaffer function reaches the global maximum value 1 at point $(0,0)$ but we define 0.9975 as the criteria situation in the Genetic Algorith m and proposed Chaos Genetic Algorithm (CGA). Other parameters of Genetic Algorithm such as population size, probability of crossover, etc. have been introduced below. Figure 6 (a) show the results of GA and proposed CGA on the Schaffer function.

- Population Size $=40$;

- Probability Crossover $=0.6$;

- Probability Mutation = 0.02;

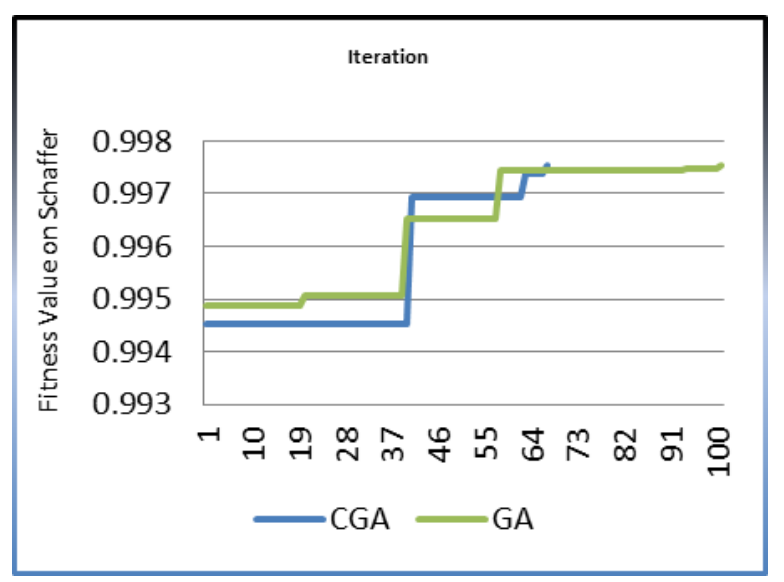

(a)

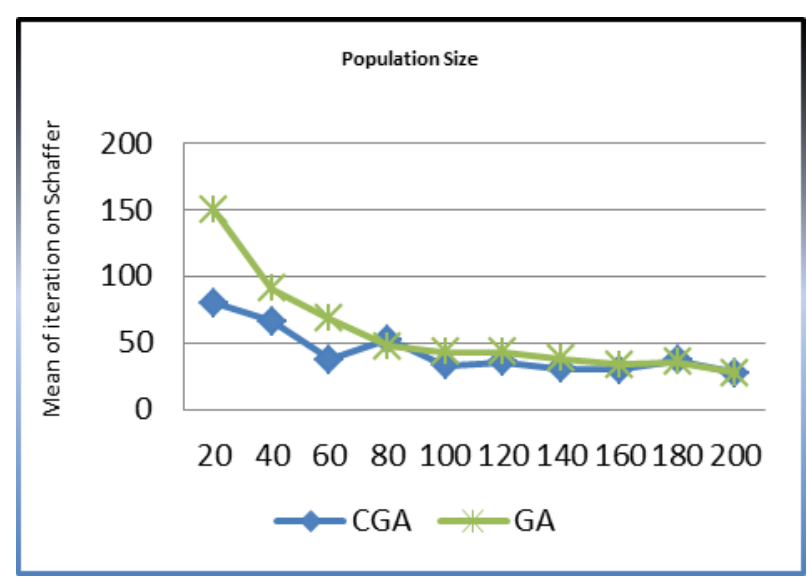

(b)

Fig. 6: (a) The GA and CGA criteria satisfaction on Schaffer function, (b) Decreasing of time complexity when Population size is increase

As it is sensible in the Figures 6 and 7, the result of CGA based on Lorenz chaotic system on the Schaffer function is much more efficient than basic GA. The result of time complexity also decreases with increasing population size in GA and CGA. Figure 6 (b) shows the effect of growing population size in GA and CGA on the Schaffer function. We have tested the above method on the Clonalg function and the results were as well as Schaffer function, figure 7 shows my experience on Clonalg function. 


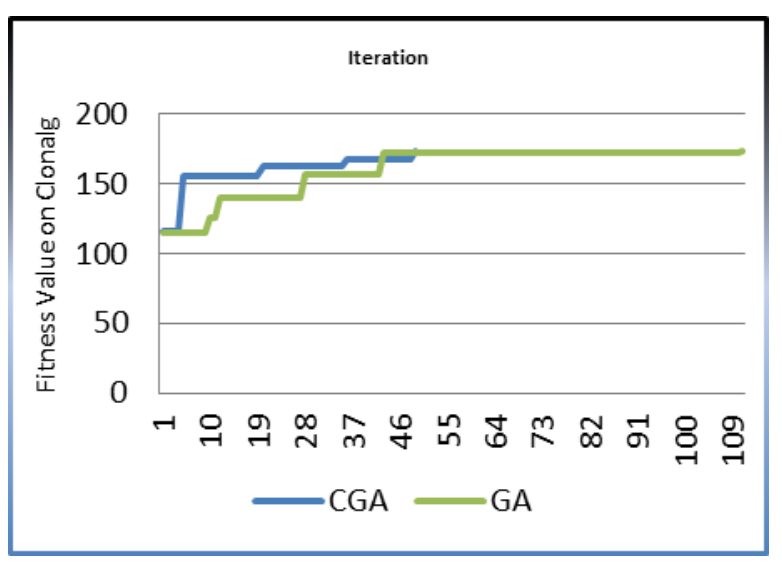

(a)

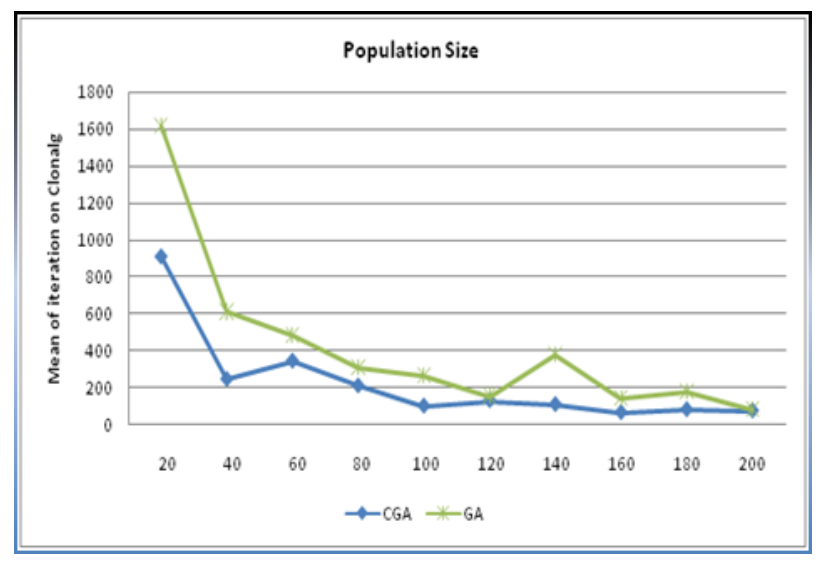

(b)

Fig. 7: (a) The GA and CGA criteria satisfaction on Clonalg function, (b) Decreasing of timecomplexity when Population size is increase

\section{Conclusion}

In this paper, the Lorenz chaotic system was used instead randomness in the basic Genetic Algorithm. We introduced a hybrid method which uses a chaotic system that can avoid premature convergence. The proposed chaotic system is Lorenz, which is a famous three dimension dynamic chaotic system. The results achieved in this experience show that Chaos Genetic Algorithm is more efficient than the traditional Genetic Algorithm and can converge to the optimum solution rapidly. \%34 improvement in Schaffer function and \%54 improvement in Clonalg function are two sample results of our method that achieved by Lorenz Chaotic Genetic Algorithm proposed in this paper. The results of our implementation show improving of Chaos Genetic Algorithm instead traditional Genetic Algorithm but there are some other famous chaotic systems such as Henon map, Logistic map, Rossler map, Tent map, Zaslavskii map, etc. that can be used instead Loren z system and also evaluate their results to find out the most efficient one.

\section{Acknowledgements}

Authors would like to thank Professor Axle Pin z for his support; moreover this work was partly funded by Islamic Azad University of Zahedan, Iran.

\section{References}

[1] Ioannis G. Tsoulos: Solving constrained optimization problems using a novel genetic algorithm. Applied Mathematics and Computation (AMC) 208(1):273-283 (2009)

[2] Wei Juan, Wang Ping: Optimization of Fuzzy Rule Based on Adaptive Genetic Algorithm and Ant Colony Algorithm. Computational and Information Sciences (ICCIS), International Conference on 359-362 (2010)

[3] Sun Feng-jie, Tian Ye: Transmission Line Image Segmentation Based GA and PSO Hybrid Algorithm. Computational and Information Sciences (ICCIS), International Conference on 677-680 (2010)

[4] Lili Liu, Qiang Zhang, Xiaopeng Wei: A RGB image encryption algorithm based on DNA encoding and chaos map. Computers \& Electrical Engineering (CEE) 38(5):1240-1248 (2012)

[5] Leo Yu Zhang, Chengqing Li, Kwok-Wo Wong, Shi Shu, Guanrong Chen: Cryptanalyzing a chaos based image encryption algorithm using alternate structure. Journal of Systems and Software (JSS) 85(9):2077-2085 (2012)

[6] Xingyuan Wang, Lin Teng, Xue Qin: A novel colour image encryption algorith $m$ based on chaos. Signal Processing (SIGPRO) 92(4):1101-1108 (2012)

[7] A. Kanso, M. Ghebleh. A fast and efficient chaosbased keyed hash function. Communications in Nonlinear Science and Numerical Simulation, 18 (1) 109:123 (2013)

[8] Hu Yuxia, Zhang Hongtao. Chaos Optimization Method of SVM Parameters Selection for Chaotic Time Series Forecasting. Physics Procedia, 25 (1) 588:594 (2012)

[9] Qinglan Lia, Pengcheng Xu. Estimation of Lyapunov spectrum and model selection for a chaotic time series. Applied Mathematical Modelling, 36 (12) 6090:6099 (2012)

[10] M. Eisencraft' R.D. Fanganiello, J.M.V. Grzybowski, D.C. Soriano, R. Attux, A.M. Batista. Chaos-based communication systems in non-ideal channels. Communications in Nonlinear Science and Numerical Simulation, 17 (12) 4707:4718 (2012)

[11] Ming-Yuan Cheng and Kuo-Yu Huang, Genetic Algorithm-Based Chaos Clustering approach for 
nonlinear optimization. Journal of Marine Science and Technology, Vol 18, No. 3, pp. 435:441 (2010)

[12] Huimin Jiang, C.K. Kwong, Zengqiang Chen, Y.C. Ysim. Chaos particle swarm optimization and $\mathrm{T}-\mathrm{S}$ fuzzy modeling approaches to constrained predictive control. Expert Systems with Applications, 39 (1) 194:201 (2012)

[13] Amir Hossein Gandomi, Gun Jin Yun, Xin-She Yang, Siamak Talatahari. Chaos-enhanced accelerated particle swarm optimization. Communications in Nonlinear Science and Numerical Simulation, 18 (2) 327:340 (2013)

[14] Manuel Martínez, Sergio García-Nieto, Javier Sanchis, Xavier Blasco Ferragud: Genetic algorithms optimization for normalized normal constraint method under Pareto construction. Advances in Engineering Software (AES) 40(4):260-267 (2009)

[15] Gao Ye, Zheng Tao: Improved genetic algorith ms based on chaotic mutation operation and its application. Multimedia Technology (ICMT), 2010 International Conference on 1-3 (2010)

[16] Mohammad Saleh Tavazoei, Mohammad Haeri: An optimization algorithm based on chaotic behavior and fractal nature. Journal of Computational and Applied Mathematics 206 (2) 1070-1081 (2007)

[17] Qigui Yang, Caibin Zeng: Chaos in fractional conjugate Lorenz system and its scaling attractors. Commun Nonlinear Sci Numer Simulat 15 (12) 4041-4051 (2010)

[18] Awad El-Gohary, A mmar Sarhan: Optimal control and synchronization of Lorenz system with complete unknown parameters. 30 (5) 1122-1132 (2005)

[19] Lifang Yu, Yao Zhao, Rongrong Ni, Ting Li: Improved Adaptive LSB Steganography Based on Chaos and Genetic Algorithm. EURASIP J. Adv. Sig. Proc. (EJASP) 2010 (2010)

\section{Authors' Profiles}

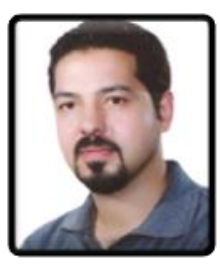

Reza Ebrahimzadeh is a faculty member of Computer Engineering at Islamic Azad University of Zahedan (IAUZAH). He got his B.S. and M.Sc. in Computer Science and Artificial Intelligence both from Computer Science Department at Islamic A zad University of Mashhad in 2006 and 2009 in order. He is now working on Biometric and Optimization laboratory of IAUZAH. His research interests include Biometrics Systems such as Iris Identification, Fingerprint Recognition, Signature Verification, Nonlinear Optimization problems and Evolutionary Computation.

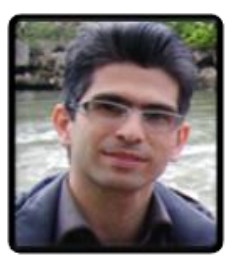

Mahdi Jampour is $\mathrm{Ph}$. D. student in Institute for Computer Graphics and Vision (ICG) at Graz University of Technology (Graz, Austria). He got his B.S. in Computer Science from Shahid Bahonar University of Kerman in 2006 and got M.Sc. from Islamic Azad University of Mashhad in Artificial Intelligence (2009). He has received a scholarship from Iran Ministry of Science, Research and Technology to continue his studies towards Ph.D. (2010). His research interests are Computer Vision and Application of Chaos theory. He is a member of IEEE and IACSIT and also editorial member of some international journals.

How to cite this paper: Reza Ebrahimzadeh, Mahdi Jampour,"Chaotic Genetic Algorithm based on Lorenz Chaotic System for Optimization Problems", International Journal of Intelligent Systems and Applications(IJISA), vol.5, no.5, pp.19-24, 2013.DOI: $10.5815 /$ ijisa.2013.05.03 